\title{
Modelling and simulation of mid-spatial- frequency error generation in CCOS
}

Bo Zhong ${ }^{1,2^{*}}$, Hongzhong Huang ${ }^{1}$, Xianhua Chen ${ }^{2}$, Wenhui Deng ${ }^{2}$ and Jian Wang ${ }^{2}$

\begin{abstract}
Background: The computer-controlled optical surfacing (CCOS) technology, which has advantages of high certainty and high convergence rate for surface error correction, has been widely applied in the manufacture of large-aperture optical elements. However, due to the convolution effect, the mid-spatial-frequency (MSF) errors are difficult to be restrained in CCOS.

Methods: Consequently, this paper presents a theoretical and experimental investigation on the generation of MSF errors, aiming to reveal its main influencing factors, and figure out the optimized parameters and the controlling strategies for restraining MSF errors. A surface topography simulation model for the generation of MSF errors was established first. Based on which, orthogonal simulation experiments were designed and conducted for the following three parameters, i.e., tool influence function (TIF), path type, and path spacing. Subsequently, the proposed model was verified through the practical polishing experiments.
\end{abstract}

Results and conclusions: The results demonstrated the influencing degree of the parameters and the optimized combination of parameters, and provided process guidance for restraining MSF errors in CCOS.

Keywords: Mid-spatial-frequency error, Convolution effect, Residual ripple, Power spectral density

\section{Background}

Ultra-precision optical elements have high machining accuracy, and play very important roles in advanced optical systems, such as, the large astronomical telescope, the extreme ultraviolet lithography and the high power laser device [1-3]. These optical systems have put forward the higher requirements (i.e., MSF errors, surface roughness, and surface defects) of optical elements than those in the traditional optical systems. Consequently, in order to achieve high performance optics in advanced optical systems, various sub-aperture polishing technologies have been well developed, including Magnetorheological
Finishing (MRF) [4], Ion Beam Figuring (IBF) [5], Plasma Jet Machining (PJM) [6] and Bonnet Polishing (BP) [7-9], etc.

Under the computer controlling, the "small tool" polishes the optics with the planned path, and the dwell time of each dwell point along the path which is determined by the surface error. Compared with the traditional manual polishing process, the CCOS process has high certainty and convergence rate for surface error correction, and does not depend on the experience of workers. However, when the small tool moves along the planned path, the ripples generated by the repeated superposition of TIF on the adjacent

\footnotetext{
* Correspondence: zhongbo_foerc@163.com

${ }^{1}$ School of Mechanical and Electrical Engineering, University Of Electronic

Science And Technology Of China, Chengdu 610054, China

${ }^{2}$ Research Center of Laser Fusion, China Academy of Engineering Physics,

Mianyang 621900, China
} 

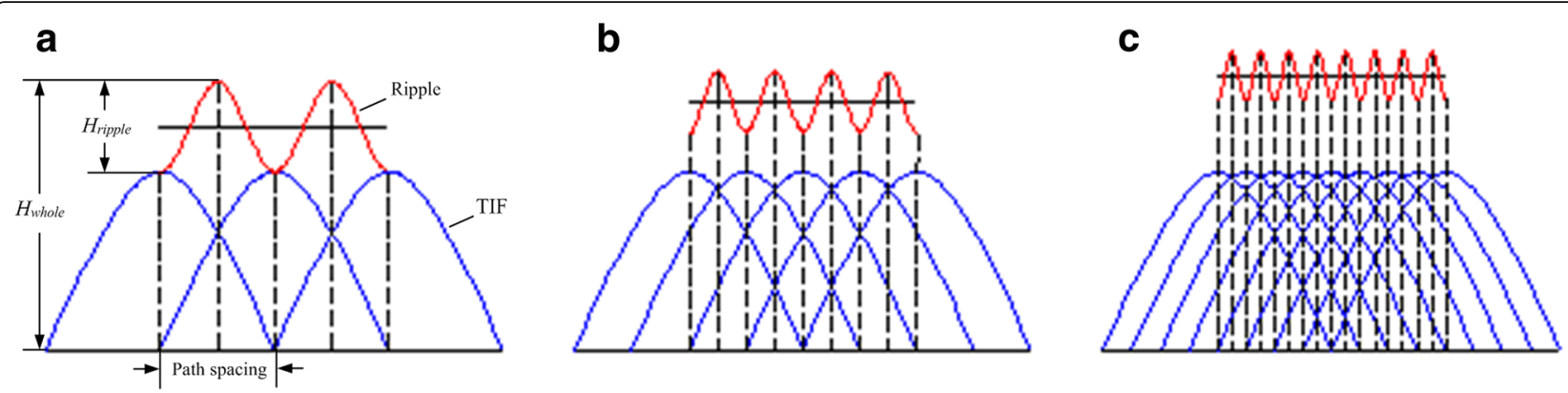

Fig. 1 Sketch map of residual ripple generation with different path spacings, (a) 0.5, (b) 0.25 and (c) 0.125 of TIF diameter

dwell points, which is known as the convolution effect. The convolution effect is a widespread phenomenon in CCOS process by using small tools, resulting in the small scale ripples.

According to National Ignition Facility Project (NIF), the small scale ripples with the spatial period between 0.12 and $33 \mathrm{~mm}$ are defined as the MSF errors. The researchers in the Lawrence Livermore National Laboratory (LLNL) found that the periodic structure induces the disturbance of amplitude or phase of laser beam through theoretical and experimental methods, which will destroy the performance of high power optical system [10, 11]. In order to ensure the beam quality and output energy of NIF laser system, NIF proposed the requirements of optical components in the MSF errors, that PSD-1:A $\leq$ $1.01 v^{-1.55}, \mathrm{RMS} \leq 1.8 \mathrm{~nm}, \mathrm{PSD}-2: \mathrm{A} \leq 1.01 v^{-1.55}, \mathrm{RMS} \leq$ $1.1 \mathrm{~nm}[10]$.

In order to restrain the MSF error, the academicians have carried out by plenty of researches. Pohl et al. [12] observed the simulation of the mid-spatial frequencies generation during the grinding process of optical components. Wang et al. [13-15] analyzed the optimization of parameters for bonnet polishing based on the minimum residual error method. In addition, a maze path was proposed, which can increase randomness while ensuring uniform trajectory distribution, and avoid periodic surface error. Wang et al. [16] have improved the removal contour of jet polishing by path offset, and combined with Hilbert path, the MSF error has been greatly improved. Tam et al. [17, 18] studied the generation algorithm of Peano path, and pointed out that the path with the variational processing direction can achieve the good removal uniformity. They also studied the influence of different tool paths (i.e., scanning path, bidirectional scanning path, Hilbert path, Peano path) on the material, and the results shown that the amplitudes of ripples under three paths (i.e., scanning path, bi-directional scanning path, Peano path) are consistent at the same path spacing. Khakpour et al. [19] proposed a path generation method applied to the abrasive jet polishing process of free surface, and studied the influences of surface profile and path type on the distribution uniformity of material removal. In order to restrain the periodic texture of optics surface, Dunn and Walker [20] developed a planning algorithm of random path, which can improve the MSF error. Dai et al. [21, 22] designed the local random path based on entropy theory, and
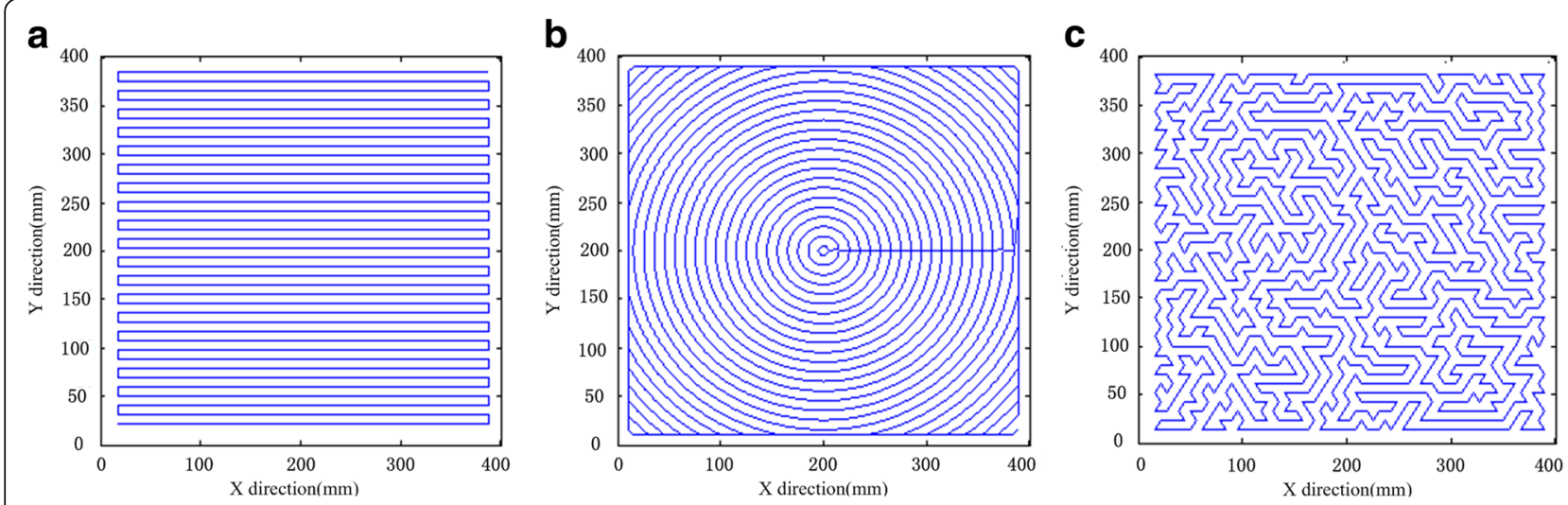

Fig. 2 Three paths, (a) raster path, (b) circle path and (c) random path 

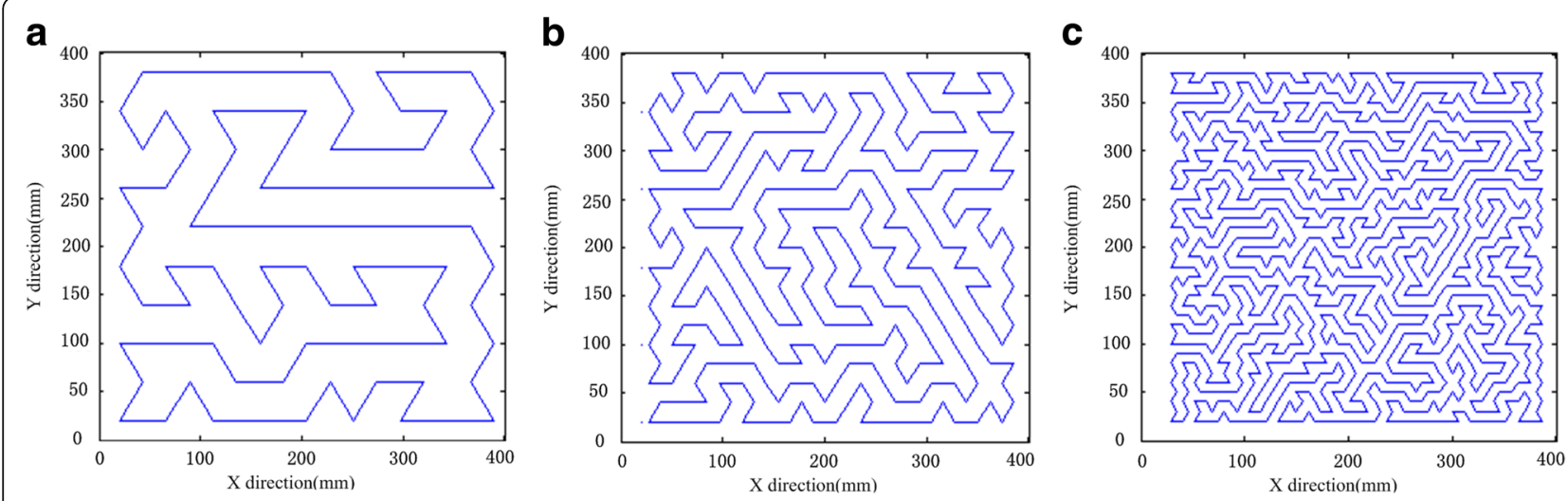

Fig. 3 Random paths generated with different path spacings, (a) $40 \mathrm{~mm}$, (b) $20 \mathrm{~mm}$ and (c) $10 \mathrm{~mm}$

proposed the random spacing of tool path based on the surface error distribution, which can effectively restrain the mid and high spatial frequency errors by MRF.

To sum up, in order to reduce the convolution effect, the scholars mainly focused on the effects of path type and path spacing on the MSF error. According to the CCOS principle, the MSF error induced by the convolution effect is determined simultaneously by the parameters such as TIF, path type and path spacing. Therefore, the influence of the combination of the parameters on MSF error should be analyzed by orthogonal simulation and experiment. The research results can not only reveal the optimized combination of parameters, but also show the influence order of parameters on MSF error.

\section{Methods}

\section{Convolution effect}

In the CCOS process, the amount of removed material $(H)$ is equal to the convolution between the TIF
$(R)$ in unit time and the dwell time function $(T)$, which can be represented as,

$$
H=R \otimes T
$$

The initial surface $(S)$ is composed by a series of discrete points $M_{i}(i=1, \ldots, n)$. The expected material removal amount $Z(M)$ on the point $M$ of surface $S$ can be expressed as,

$$
H(M)=\sum_{j=1}^{k} R\left(R_{j} ; M\right) \cdot T\left(T_{j} ; R_{j}\right)
$$

In Eq. $2, R\left(R_{j} ; M\right)$ is the removal amount of TIF $R_{j}$ in the unit time on point $M, T\left(T_{j} ; R_{j}\right)$ is the dwell time of TIF $R_{j}$, and $k$ is the number of dwell points on which TIF have removal effect on point $M$.

Figure 1 illustrates the sketch map of residual ripple generation when the dwell time of all points on the surface is equal. In Fig. 1, $H_{\text {ripple }}$ is the residual ripple amplitude, and $H_{\text {whole }}$ is the total removal depth. Comparing

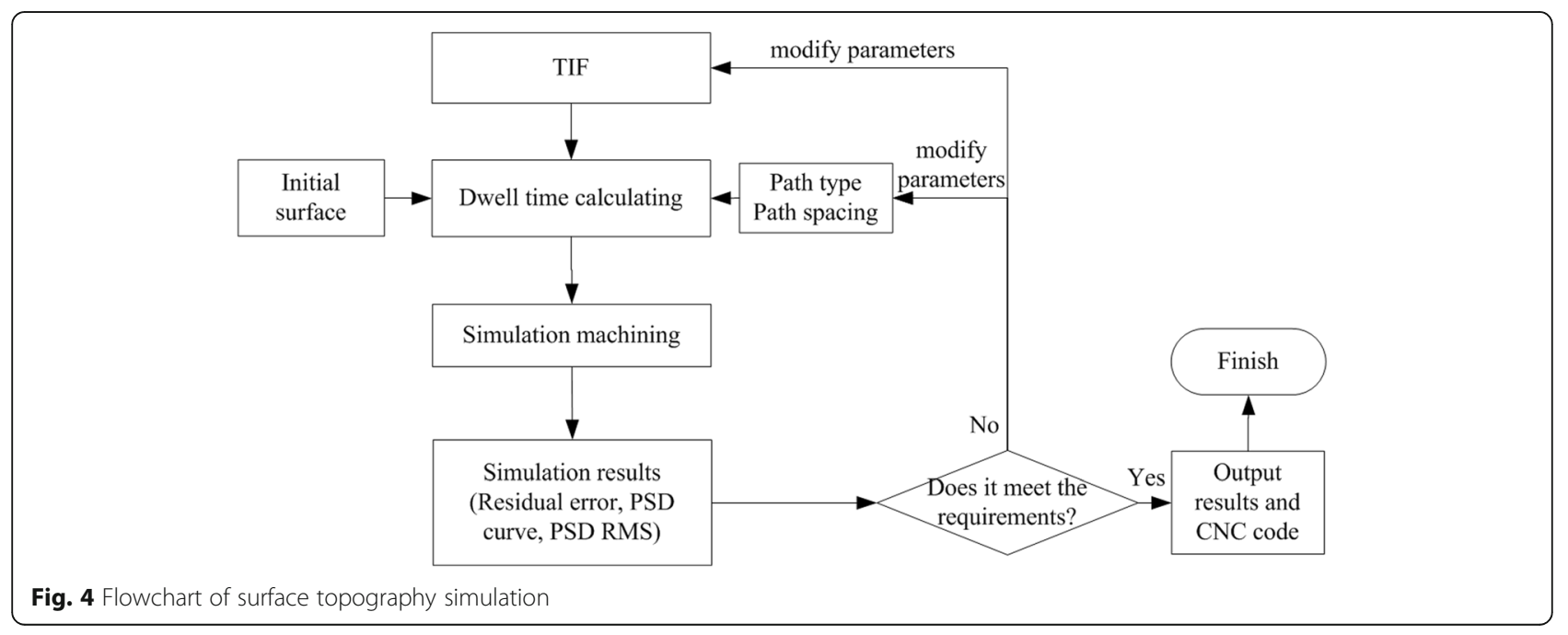




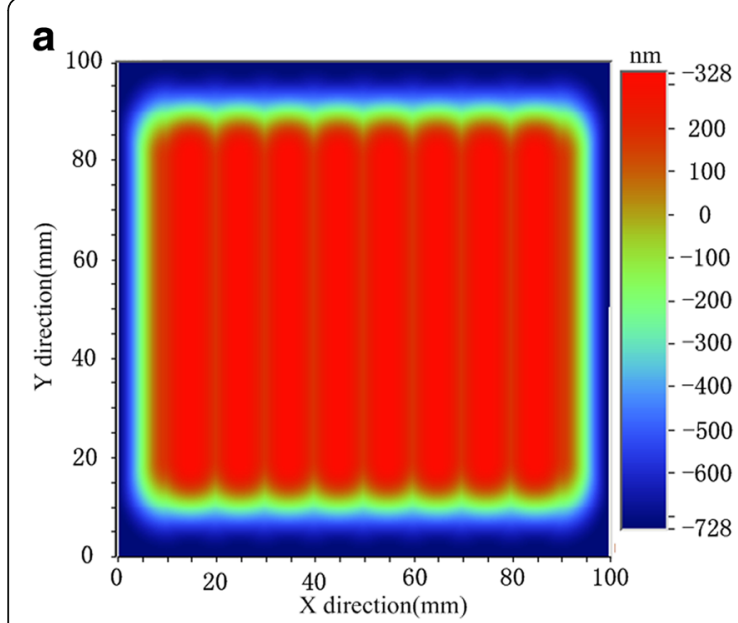

b

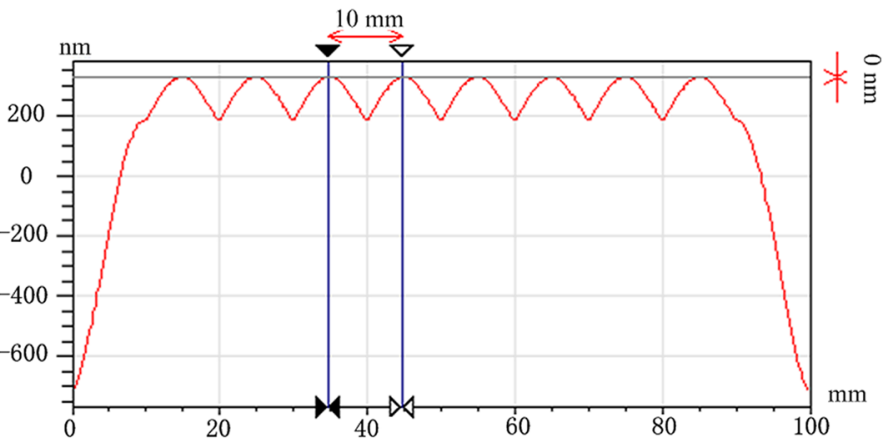

Fig. 5 a The residual error map after the simulation and $\mathbf{b}$ the contour in the $X$ direction

Fig. 1(a)-(c), the $H_{\text {whole }}$ increases as the path spacing increases, while the $H_{\text {ripple }}$ decreases.

\section{Path generation}

According to the CCOS principle, the path has marked influence on the MSF errors. Therefore, in this section the generation methods of various paths (i.e., raster path, circle path, random path) are studied, and lay the foundation for the following experiments.

The flow of generating various paths mainly includes the definition of variables, the definition of variable initial value, the path selection, the calculation of dwell point coordinates $\left(x_{p}, y_{p}\right)$, the boundary condition, and the output of dwell point coordinates. It should be pointed out that the main differences of three paths are the calculation formulas of dwell point coordinates, and the compendiary calculation formulas for raster path, circle path and random path are the Eqs. 3-5, respectively. Figure 2 shows the three paths that have been implemented.

$$
\left\{\begin{array}{l}
x_{p}=x_{p-1}+\text { Step or } x_{p}=x_{p-1}-\text { Step } \\
y_{p}=y_{p-1}+\text { PathSpacing }
\end{array}\right.
$$
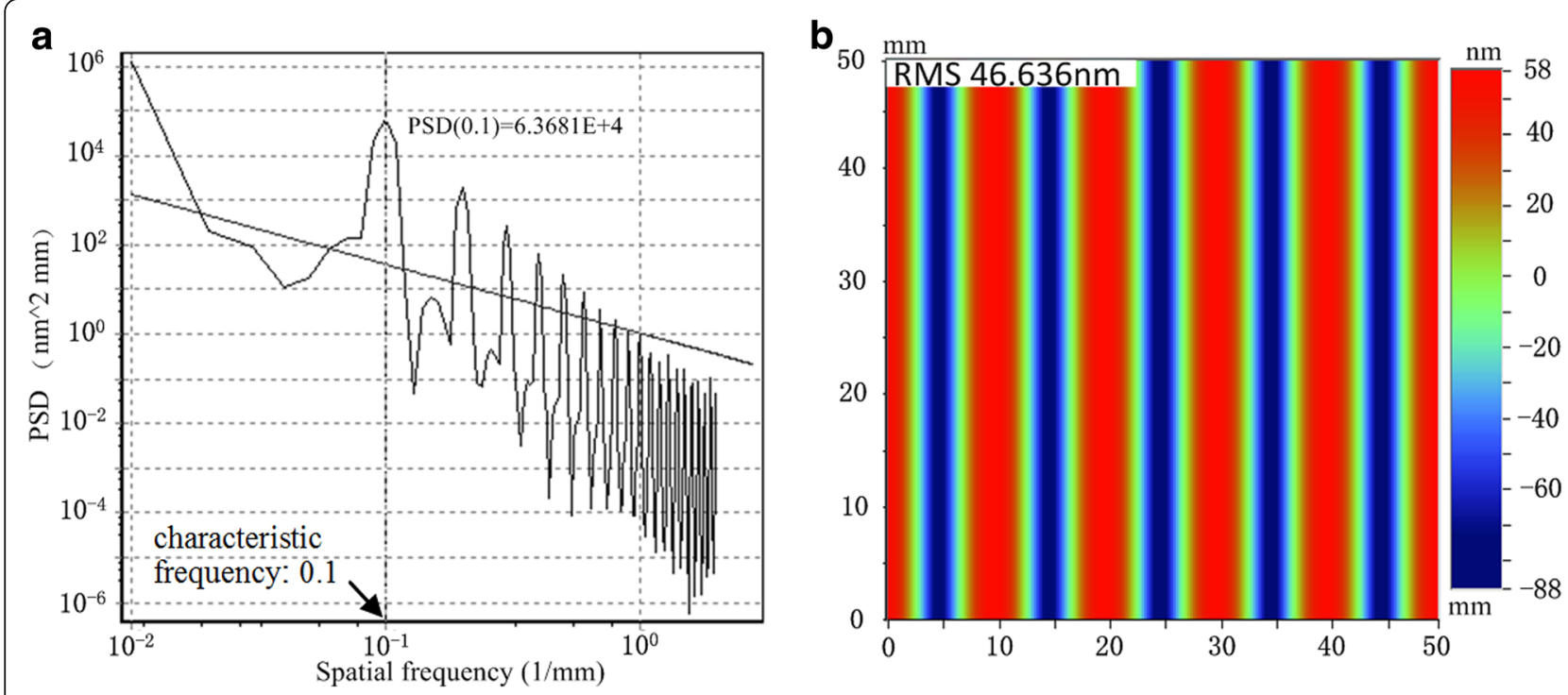

Fig. 6 a The PSD curve of residual error and $\mathbf{b}$ the residual ripple distribution $(50 \mathrm{~mm} \times 50 \mathrm{~mm})$ 

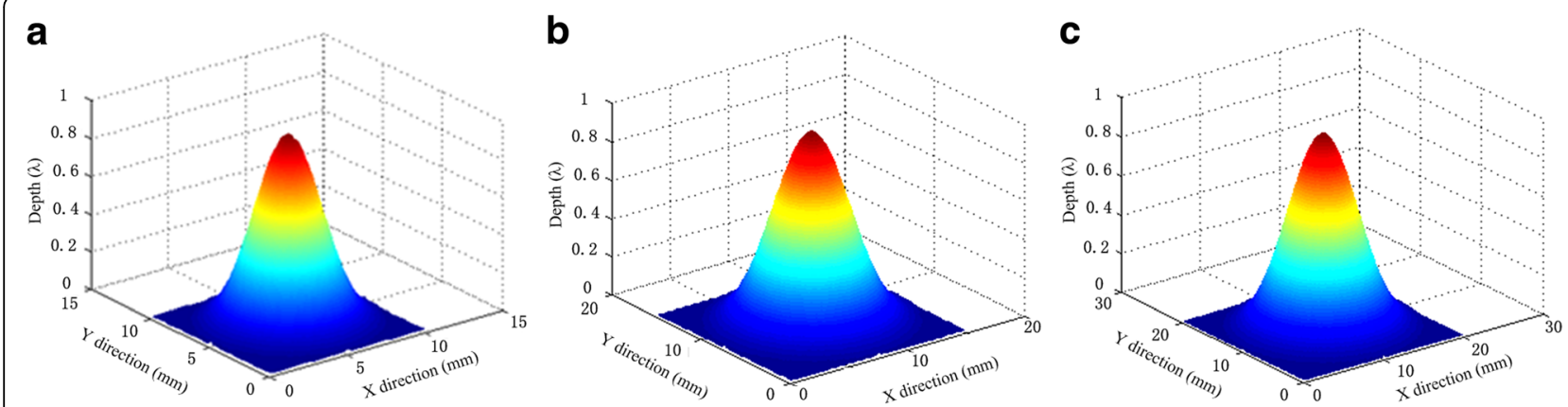

Fig. 7 Theoretical TIFs with differet radiuses, (a) $5 \mathrm{~mm}$, (b) $7.5 \mathrm{~mm}$ and (c) $10 \mathrm{~mm}$

$$
\begin{aligned}
& \left\{\begin{array}{l}
x_{p}=r \cdot \cos (\text { Angle }) \\
y_{p}=r \cdot \sin (\text { Angle }) \\
r=r_{0}: \text { PathSpacing }: r_{n} \\
\text { PointNumber }=2 \cdot \text { pi } \cdot r / \text { Step } \\
\text { Angle }=0: 2 \cdot \text { pi } / \text { PointNumber }: 2 \cdot \text { pi }
\end{array}\right. \\
& \left\{\begin{array}{l}
x_{p}=x_{p-1}+d x \cdot \cos (\text { Angle }(i)) \\
y_{p}=y_{p-1}+d x \cdot \sin (\text { Angle }(i)) \\
d x=(\text { PathSpacing } / \text { sqrt }(3)) \cdot 2 \\
\text { Angle }=\text { pi } / 3: \text { pi } / 3: 2 \cdot \text { pi } \\
i=\text { randperm }(6,1)
\end{array}\right.
\end{aligned}
$$

In Eqs. 3-5, $\left(x_{p}, y_{p}\right)$ are the coordinates of the dwell point, Step is the distance of adjacent dwell points on the path, Path spacing is the distance of adjacent path, $r_{0}$ is the starting radius, $r_{n}$ is the ending radius, and $i$ is the sequence number of random direction. The random path has different travel directions, and the each travel direction selects by the random number $i$.

Figure 3 shows random paths with different path spacings. For workpieces of same size, the path spacing changes the density degree of path. The path spacing is small and the path is dense. From a dense random path, the path seems more random and may help improve the surface texture, which will be validated in subsequent analysis. It should note that the path generated each time is totally different with another, as shown in Fig. 3. This reflects the randomness of random path.

\section{Surface topography simulation model}

Figure 4 is a flowchart of surface topography simulation. In Fig. 4, firstly, enter the initial surface, the TIF, the path type and the path spacing. Secondly, the simulating surface topography is achieved by the iterative convolution calculation. At last, the PSD curve is calculated by the power spectral density method, and the error distribution in a specified frequency band is calculated by the filter method.

Surface topography simulation model is used to calculate the residual error with the Gaussian TIF and the raster path, and the MSF error is analyzed. In the simulation, the processing area is $100 \mathrm{~mm} \times$ $100 \mathrm{~mm}$, the TIF diameter is $20 \mathrm{~mm}$, and the path spacing is $10 \mathrm{~mm}$. Figure 5 shows the residual error map after the simulation and the contour in the $\mathrm{X}$ direction. Figure 6 illustrates the PSD curve of residual error and the residual ripple distribution (50 $\mathrm{mm} \times 50 \mathrm{~mm})$.

It can be seen from Fig. 5 that there is a regular raster ripple (period $10 \mathrm{~mm}$ ) consistent with the path spacing $(10 \mathrm{~mm})$ due to the effect of the convolution effect in the raster path simulation process, and the PSD curve produces a significant peak (shown in the red circle position in Fig. 6(a)), whose frequency is $0.1 \mathrm{~mm}^{-1}$. This paper defines the frequency at a significant peak in the PSD curve as the characteristic frequency, whose amplitude is the PSD value of characteristic frequency (PSDc). Figure 6(b) shows the residual ripples in the middle region $(50 \mathrm{~mm} \times 50 \mathrm{~mm})$ of the residual error. In this paper, the root-mean-square of residual ripple in the $50 \mathrm{~mm} \times 50 \mathrm{~mm}$ region is defined as the RMS value of residual ripple (RMSr). In the following orthogonal simulation and experiment, the PSDc and the $\mathrm{RMSr}$ are used to evaluate the effect of restraining the MSF errors.

Table 1 Level of each factor in orthogonal simulation experiment

\begin{tabular}{lllll}
\hline Factor & & $\begin{array}{l}\text { TIF diameter } \\
\mathrm{mm}\end{array}$ & Path type & $\begin{array}{l}\text { Path spacing } \\
\mathrm{mm}\end{array}$ \\
\hline Level & 1 & 20 & raster & 4 \\
& 2 & 15 & circle & 3 \\
& 3 & 10 & random & 2 \\
\hline
\end{tabular}


Table 2 Simulative conditions and results

\begin{tabular}{|c|c|c|c|c|c|}
\hline No. & $\begin{array}{l}\text { TIF } \\
\text { diameter } \\
\mathrm{mm}\end{array}$ & Path type & $\begin{array}{l}\text { Path } \\
\text { spacing } \\
\mathrm{mm} \\
\end{array}$ & $\begin{array}{l}\text { RMSr } \\
\mathrm{nm}\end{array}$ & $\begin{array}{l}\mathrm{PSDC} \\
\mathrm{nm}^{2} \cdot \mathrm{mm}\end{array}$ \\
\hline 1 & 20 & raster & 4 & 1.707 & 69.12 \\
\hline 2 & 20 & circle & 3 & 1.273 & 2.5 \\
\hline 3 & 20 & random & 2 & 0.958 & 1.19 \\
\hline 4 & 15 & raster & 3 & 1.713 & 62.77 \\
\hline 5 & 15 & circle & 2 & 1.665 & 1.08 \\
\hline 6 & 15 & random & 4 & 15.09 & 13.5 \\
\hline 7 & 10 & raster & 2 & 1.845 & 69.54 \\
\hline 8 & 10 & circle & 4 & 17.265 & 1700.3 \\
\hline 9 & 10 & random & 3 & 30.5 & 14.2 \\
\hline$k 1$ & 1.31 & 1.76 & 11.35 & \multirow{4}{*}{\multicolumn{2}{|c|}{$\begin{array}{l}\text { RMSr as the } \\
\text { evaluation index }\end{array}$}} \\
\hline$k 2$ & 6.16 & 6.73 & 11.16 & & \\
\hline k3 & 16.54 & 15.52 & 1.49 & & \\
\hline Range & 15.22 & 8.78 & 9.86 & & \\
\hline$p 1$ & 24.27 & 67.14 & 594.31 & \multirow{3}{*}{\multicolumn{2}{|c|}{$\begin{array}{l}\text { PSDc as the } \\
\text { evaluation index }\end{array}$}} \\
\hline p2 & 25.78 & 567.96 & 26.49 & & \\
\hline p3 & 594.68 & 9.63 & 23.94 & & \\
\hline Range & 570.41 & 558.33 & 570.37 & & \\
\hline
\end{tabular}

\section{Conditions and results}

\section{Orthogonal simulation experiments}

Three main factors, the TIF diameter, the path type and the path spacing, are considered in orthogonal simulation. The TIF is assumed to be the Gaussian type (shown in Fig. 7). The path types include raster path, circle path, and random path with path spacing of 2,3 and $4 \mathrm{~mm}$. In addition, the error of initial surface is 0 , and its size is $100 \mathrm{~mm} \times 100 \mathrm{~mm}$. To ensure the comparability of results, the simulation results are normalized. The RMSr and the PSDc under unit removal depth are used as evaluative indexes, meanwhile the RMSr and PSDc are calculated within the band filter for $0.5 \mathrm{~mm}-10 \mathrm{~mm}$. The level of each factor is shown in Table 1. The simulation conditions and results are shown in Table 2. The surface simulation results under various combined parameters, including the residual error distribution $(100 \mathrm{~mm} \times 100 \mathrm{~mm})$, PSD curve, and the ripple distribution $(50 \mathrm{~mm} \times 50 \mathrm{~mm})$ are shown in Appendix 1.

In simulation experiment, the TIF is assumed to be Gaussian type, shown as follow,

$$
R(x, y)=A \cdot \exp \left[-\frac{x^{2}+y^{2}}{\left(\frac{r}{2}\right)^{2}}\right]
$$

In Eq. 6, $A$ is the adjustment factor of amplitude, and $r$ is the radius of TIF, $x \in(-r, r), y \in(-r, r)$. When $A$ is assumed as 1 and $r$ is assumed as $5 \mathrm{~mm}$,
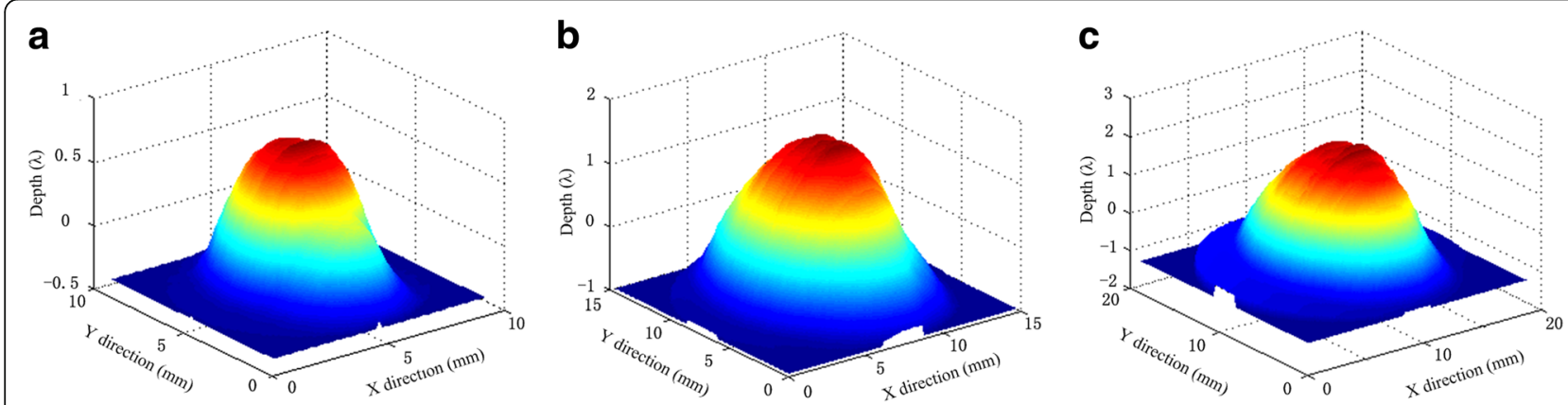

Fig. 8 Actual TIFs with differet radiuses, (a) $4.5 \mathrm{~mm}$, (b) $7.25 \mathrm{~mm}$ and (c) $9.5 \mathrm{~mm}$ 
Table 3 Experimental conditions and results

\begin{tabular}{|c|c|c|c|c|c|}
\hline No. & $\begin{array}{l}\text { TIF } \\
\text { diameter } \\
\mathrm{mm}\end{array}$ & Path type & $\begin{array}{l}\text { Path } \\
\text { spacing } \\
\mathrm{mm} \\
\end{array}$ & $\begin{array}{l}\text { RMSr } \\
\mathrm{nm}\end{array}$ & $\begin{array}{l}\mathrm{PSDC} \\
\mathrm{nm}^{2} \cdot \mathrm{mm}\end{array}$ \\
\hline 1 & 20 & raster & 4 & 7.838 & 1384 \\
\hline 2 & 20 & circle & 3 & 3.285 & 53.43 \\
\hline 3 & 20 & random & 2 & 3.23 & 4.68 \\
\hline 4 & 15 & raster & 3 & 13.858 & 5570 \\
\hline 5 & 15 & circle & 2 & 3.274 & 10.24 \\
\hline 6 & 15 & random & 4 & 18.152 & 14.89 \\
\hline 7 & 10 & raster & 2 & 7.199 & 913 \\
\hline 8 & 10 & circle & 4 & 25.513 & 8311 \\
\hline 9 & 10 & random & 3 & 25.718 & 1955 \\
\hline k1 & 4.78 & 9.63 & 17.17 & \multirow{4}{*}{$\begin{array}{l}\text { RMSr as the } \\
\text { evaluation index }\end{array}$} & \\
\hline$k 2$ & 11.76 & 10.69 & 14.29 & & \\
\hline k3 & 19.48 & 15.70 & 4.57 & & \\
\hline Range & 14.69 & 5.01 & 12.60 & & \\
\hline$p 1$ & 480.70 & 2622.33 & 3236.63 & \multirow{4}{*}{$\begin{array}{l}\text { PSDc as the } \\
\text { evaluation index }\end{array}$} & \\
\hline$p 2$ & 1865.04 & 2791.56 & 2526.14 & & \\
\hline p3 & 3726.33 & 658.19 & 309.31 & & \\
\hline Range & 3245.63 & 2133.37 & 2927.32 & & \\
\hline
\end{tabular}

$7.5 \mathrm{~mm}, 10 \mathrm{~mm}$, the corresponding theoretical TIFs are shown in Fig. 7(a), (b), and (c), respectively.

\section{Practical polishing experiments}

In order to verify the influence of parameter combination on the MSF error further, the practical orthogonal experiments are carried out. The conditions in the practical experiments are consistent with the orthogonal simulation experiments and a selfdeveloped bonnet polishing machine was adopted. When the bonnet radius $R=80 \mathrm{~mm}$ is used, the actual TIF with the two compressions, i.e., 0.65 and $0.35 \mathrm{~mm}$, can be obtained, as shown in Fig. 8(a) and (b). And their actual radius is 4.5 and $7.25 \mathrm{~mm}$ respectively. When the bonnet radius $R=40 \mathrm{~mm}$ is used, the actual TIF with the compression $0.3 \mathrm{~mm}$ can be obtained, as shown in Fig. 8(c), and its actual radius is $9.5 \mathrm{~mm}$. The experiment conditions and results are shown in Table 3 . The practical polishing results under various combined parameters, including the residual error distribution (100 $\mathrm{mm} \times$ $100 \mathrm{~mm}$ ), PSD curve, and the ripple distribution $(50 \mathrm{~mm} \times 50 \mathrm{~mm})$ are shown in Appendix 2 .

\section{Discussion}

Influence of process parameter combination on PSDc According to Tables 2 and 3, the PSDc values in the simulation and practical experiments are drawn in Fig. 9.

Figure 9 shows that each factor has the same tendency to the evaluative index (PSDc) in simulation and experiment. However, it is necessary to point
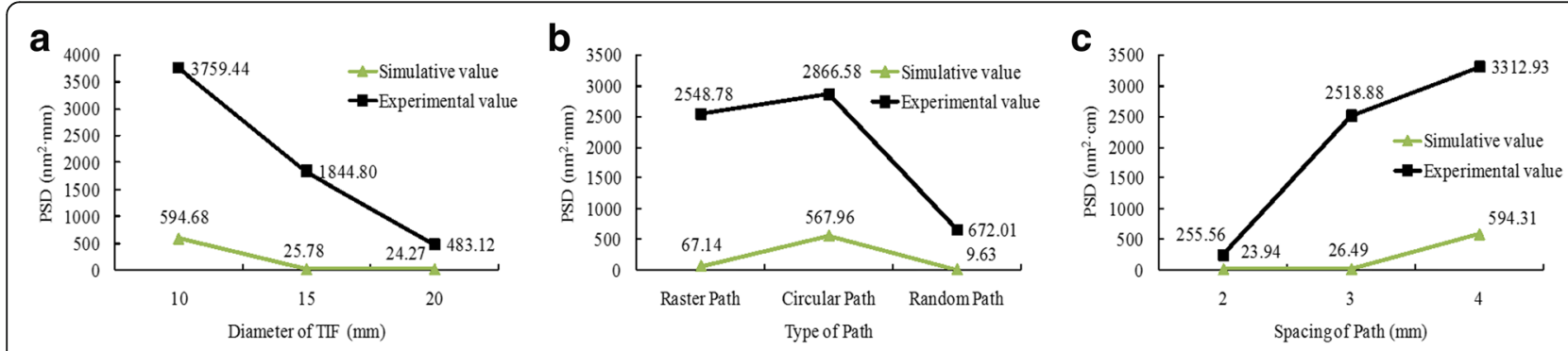

Fig. 9 Influence of various factors on PSDc, (a) TIF diameter, (b) path type and (c) path spacing 


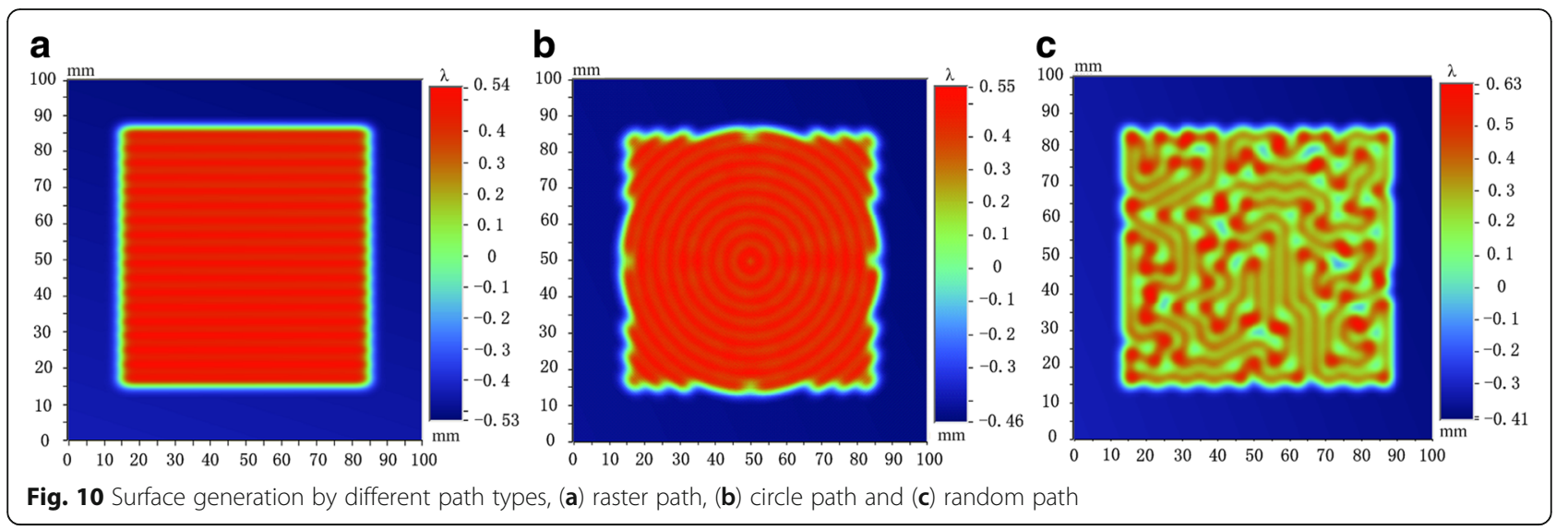

out that the simulation results under each set of parameters are smaller than the experimental results. The possible reason is that there is a difference between the simulated and actual polishing process. The simulation is based on the TIF of Gaussian type and the linear removal model, while in the polishing process, the TIF has the shape distortion influenced by the instability of polishing conditions, i.e., polishing fluid, tool condition and polishing environment. Actually, the TIF is time-varying in the practical polishing process.

According to the orthogonal simulation and experimental results, the influence of process parameters on the PSDc from three aspects are discussed in this section.

(1)Influencing degree of the parameters: in Tables 2 and 3 , it shows that the ranges of PSDc values of TIF diameter in the simulation and practical experiments are maximal, and their value are $570.41 \mathrm{~nm}^{2} \cdot \mathrm{mm}$, $3245.63 \mathrm{~nm}^{2} \cdot \mathrm{mm}$, respectively. While the ranges of PSDc values of path type in the simulation and practical experiments are minimal, and their value are 558.33 and $2133.37 \mathrm{~nm}^{2} \cdot \mathrm{mm}$. According to the ranges of PSDc of parameters, it is shown that the parameters in descending order of impact are as follows: TIF diameter, path spacing, and path type.

(2) Optimal combined parameters: according to Fig. 9, the simulation and experimental results show that the combined parameters, i.e., $20 \mathrm{~mm}$ of TIF diameter, $2 \mathrm{~mm}$ of path spacing, and random path, can obtain the minimal PSDc, which is considered to be an optimal combination in the selected parameters.

(3) Relationship between parameters and evaluative index: it can be seen from Fig. 9, the influences of parameters on the evaluative index (PSD value) is that, the larger the TIF diameter is, and the smaller the path spacing is, the smaller the PSDc is, otherwise the path types in descending order of impact are as follows: random path, raster path, and circle path. Figure 10 shows the surface generation by different path types, i.e., raster path, circle path and random path. According to Fig. 10, the PSD curves by three path types are calculated, shown in Fig. 11.

Orthogonal analysis determines that the random path has the optimal ability to restrain the PSDc. The random path is an unicursal path with three random direction angles (i.e., $60^{\circ}, 120^{\circ}$, and $180^{\circ}$ ). In the processing process, it will produce the ripples in three directions, i.e., $60^{\circ}, 120^{\circ}$, and $180^{\circ}$. Although the random path also has a certain regularity, which is slighter than the raster and circle path, so the regular ripple will be suppressed, that is, the PSDc consistent with the path spacing is suppressed.

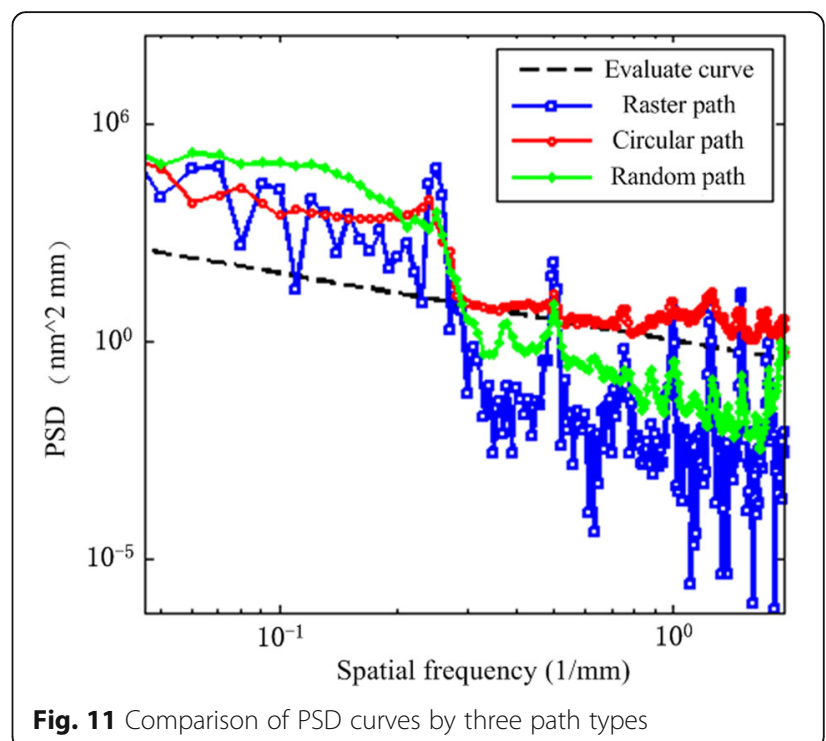



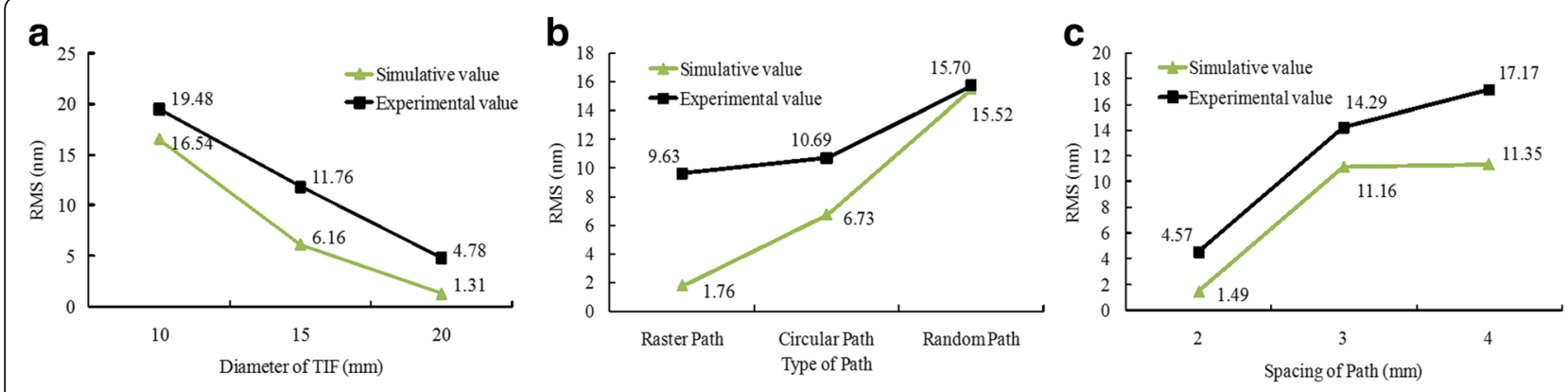

Fig. 12 Influence of various factors on RMSr, (a) TIF diameter, (b) path type and (c) path spacing

\section{Influence of process parameter combination on RMSr}

According to Tables 2 and 3, the RMSr values in the simulation and practical experiments are drawn in Fig. 12 .

Figure 12 illustrates the comparisons between the measured and the simulated results about the RMS of ripple under various conditions. It shows that the simulated ripple exhibits a good agreement with the practical measured ripple. However, the simulation results are smaller than the experimental results. The reason may be consistent with the previous analysis in "Influence of process parameter combination on PSDc" section, which is due to the deterioration of MSF error caused by the instability of processing conditions during the actual process. The dynamic factors will be investigated to improve the removal determinism in further study which is not within the scope for this paper.

According to the orthogonal simulation and experimental results, the influence of process parameters on the RMSr from three aspects are discussed in this section.

\section{(1)Influencing degree of the parameters: in}

Tables 2 and 3, it shows that the ranges of RMSr values of TIF diameter in the simulation and practical experiments are maximal, and their value are 15.22 and $14.69 \mathrm{~nm}$, respectively. While the ranges of RMSr values of path type in the simulation and practical experiments are minimal, and their value are $8.78 \mathrm{~nm}, 5.01 \mathrm{~nm}$, respectively. According to the ranges of RMSr of parameters, we can see that the parameters in descending order of impact are as follows: TIF diameter, path spacing, and path type.

(2) Optimal combined parameters: according to Fig. 12, the simulation and experimental results show that the combined parameters, i.e., $20 \mathrm{~mm}$ of TIF diameter, $2 \mathrm{~mm}$ of path spacing, and raster path, can obtain the minimal RMSr, which is considered to be an optimal combination in the selected parameters.

The optimized parameters obtained in this part are inconsistent with the "Influence of process parameter combination on PSDc" section, mainly due to the different evaluation indexes used in the two sections, which are PSDc and RMSr respectively. In the actual processing, in order to ensure that the two evaluation indexes can be qualified, the two sets of optimized parameters can be used alternately.

(3) Relationship between parameters and evaluative index: it can be seen from Fig. 12, the influences of parameters on the RMSr is that, the larger the TIF diameter is, the smaller the path spacing is and the

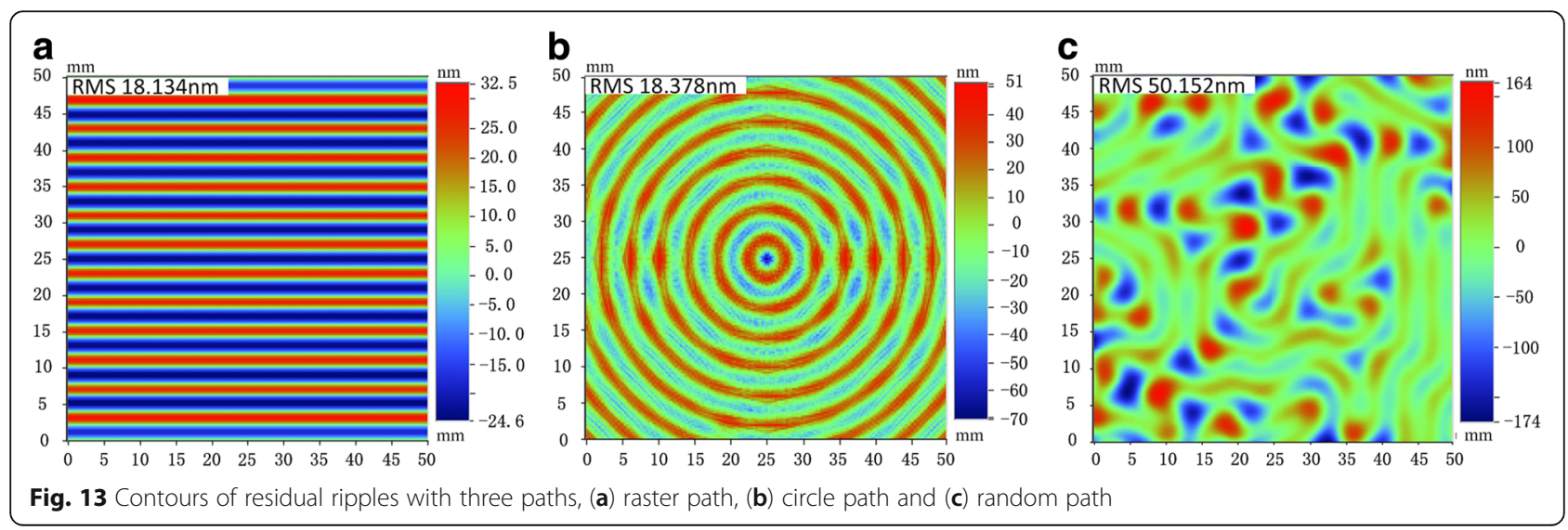




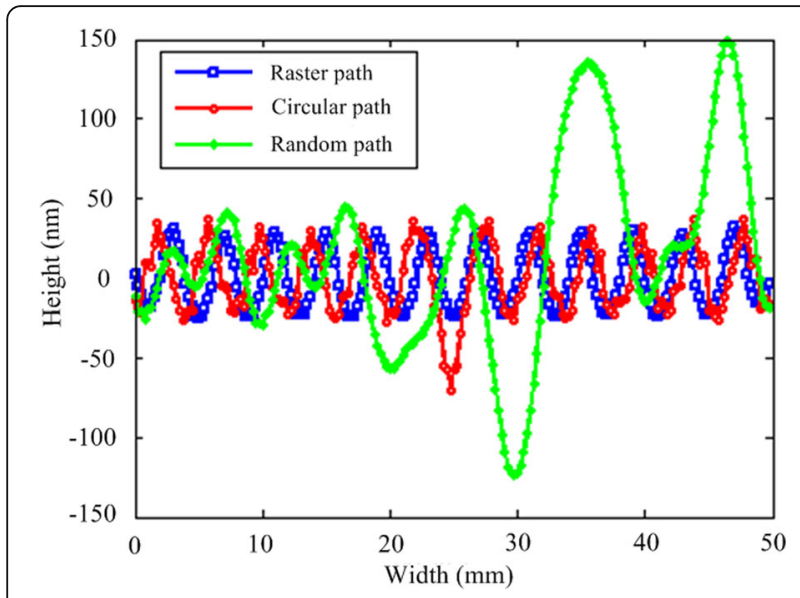

Fig. 14 Curves of residual ripples with three paths

smaller the RMSr is, otherwise the path types in descending order of impact are as follows: raster path, circle path, and random path.

The influence of TIF diameter and path spacing on the ripples is similar to the previous research results [13]. The following analysis focuses on the influence of path type on the RMSr. Figure 13 shows the contours of residual ripples with three paths, i.e., raster path, circle path, random path, with keeping other process parameters consistent. Figure 14 is the curves of residual ripples with three paths. It can be seen from Fig. 14 that the ripple amplitude of raster path is minimal, and the ripple amplitude of circle path is slightly larger than that of raster path, and the ripple amplitude of random path is maximal. According to the characters of three paths, it shows that the ripple feature is related to the turn angle on the path. When the angle between the dwell points on the path is less than $180^{\circ}$, the extra removal exhibits on the corner. Theoretically, the turn angle on the raster path is $180^{\circ}$, and the turn angle between the dwell points on the circle path is related to the radius of circle. The smaller the radius is, the more extra removal is, so the residual ripple of circle path is slightly larger than the raster path, and is prone to produce "center error" at the center of circle path. Since the turn angle is generally large (more than $90^{\circ}$ ), the extra removal produced by the circle path is not significant. There are three angles, such as $60^{\circ}, 120^{\circ}$, and $180^{\circ}$ between the dwell points on the random path. Comparing the removal depth at the corner with different angles, the removal depth with corner angle $60^{\circ}$ is the maximal, and the removal depth with corner angle $180^{\circ}$ is the minimal, as shown in Fig. 15. Since the RMSr is directly related to the amplitude of ripple, the raster path produces the minimum ripple, while the random path produces the maximum ripple.

\section{Conclusions}

A surface topography simulation model for the generation of MSF errors has been established. A model-based simulation system, which involves the theoretic TIF model, path planning methods and removal model etc., can forecast the surface topography with the various combined parameters, and export the CNC programs used in the practical polishing experiments. Orthogonal simulation experiments were designed for the following three parameters, i.e., TIF diameter, path type, and path spacing, and verified through the practical polishing experiments. The RMSr and the PSDc were used to evaluate the simulations and experiments. The two evaluative indexes are both improved with the increase of TIF diameter or the decrease of path spacing. The random path can reduce the PSDc, but worsen the RMSr, while the influences of raster path on the two evaluative indexes are opposite to those of random path. In addition, the parameters in descending order of impact on the MSF errors are as follows: TIF diameter, path spacing, and path type. Therefore, in order to control the MSF errors, the optimization of TIF diameter is the top-priority strategy.
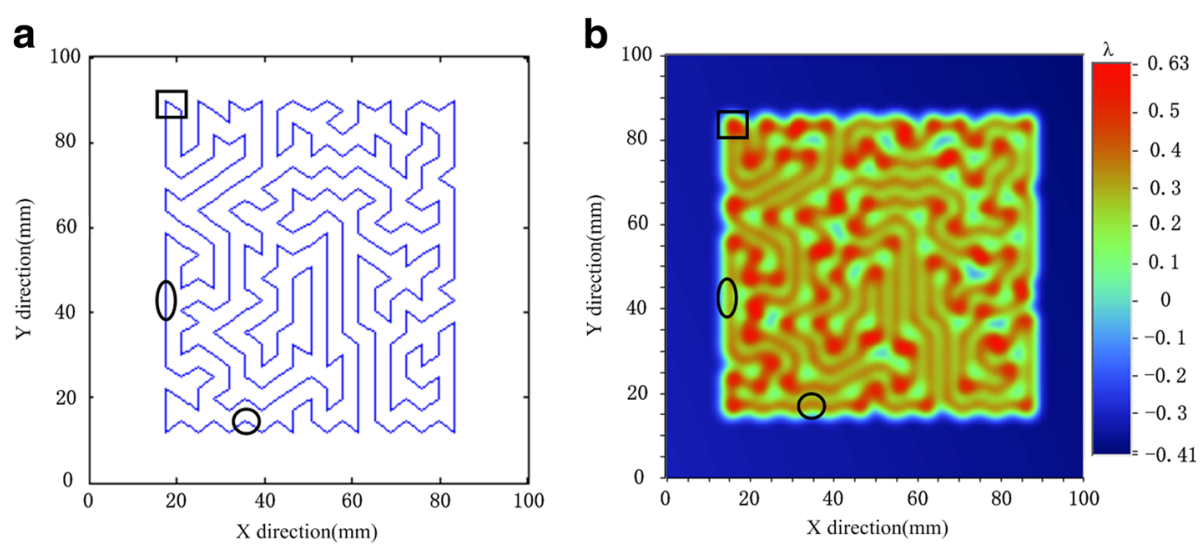

Fig. 15 Excess removal at corner of random path, (a) random path and (b) surface generation by random path 


\section{Appendix 1}

Table 4 Orthogonal Simulative results

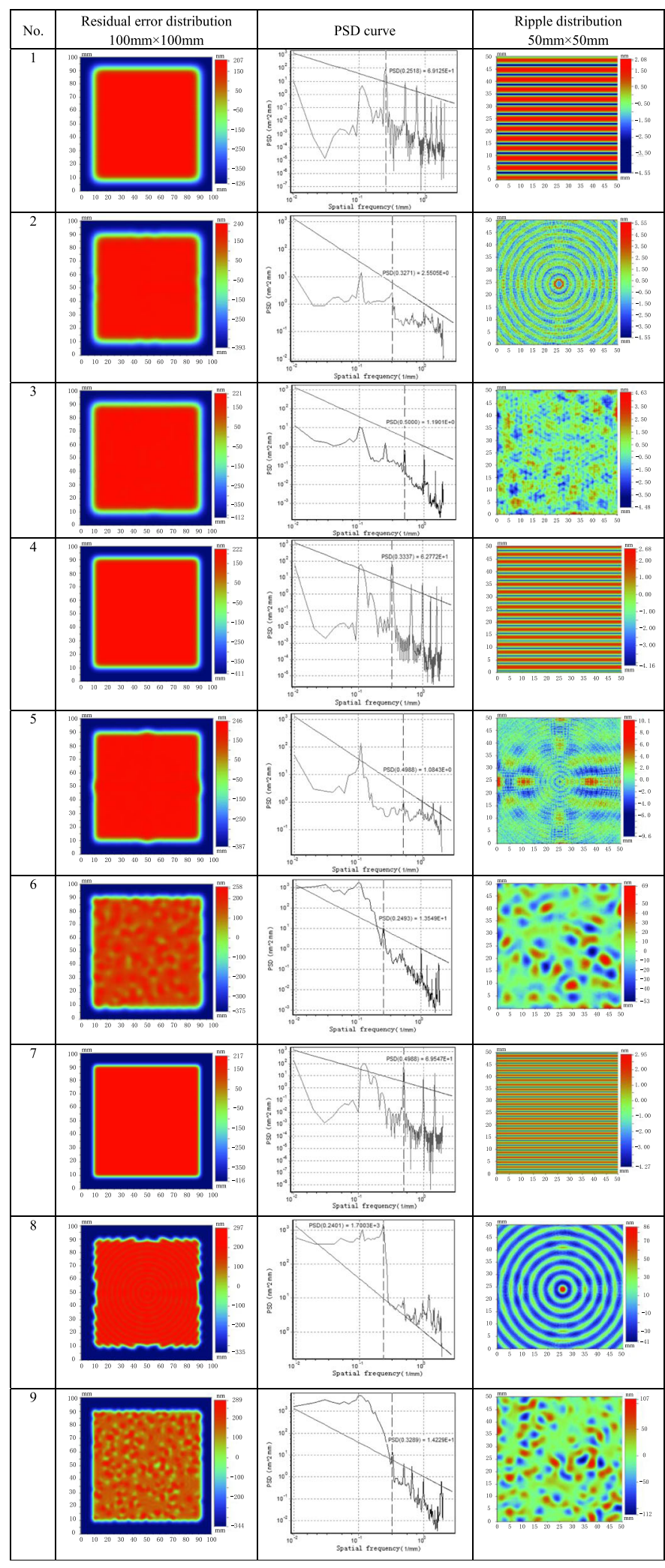




\section{Appendix 2}

Table 5 Practical polishing results

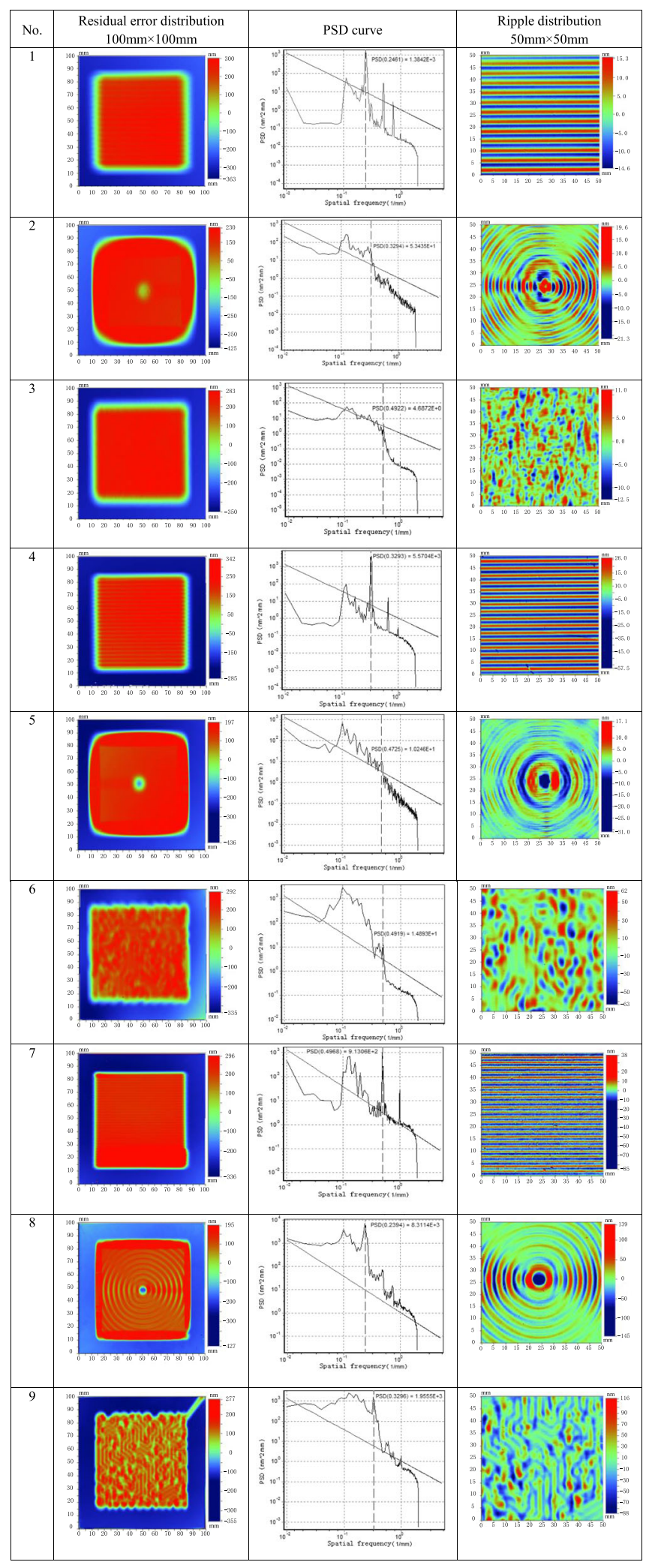




\section{Abbreviations}

BP: Bonnet Polishing; CCOS: Computer-controlled optical surfacing; IBF: Ion Beam Figuring; LLNL: Lawrence Livermore National Laboratory; MRF: Magnetorheological Finishing; MSF: Mid-spatial-frequency; NIF: National Ignition Facility Project; PJM: Plasma Jet Machining; PSD: Power spectral density; PSDc: PSD value of characteristic frequency; RMS: Root-mean-square; RMSr: RMS value of residual ripple; TIF: Tool influence function

\section{Acknowledgements}

Not applicable.

\section{Funding}

This work was financially supported by the Science Challenge Project (No. JCKY2016212A506-0502) and the Youth Talent Fund of Laser Fusion Research Center, CAEP. (No. RCFCZ1-2017-6).

Availability of data and materials

Data will be shared after publication.

\section{Authors' contributions}

BZ and $\mathrm{HH}$ developed the surface topography simulation model; XC, WD and JW assisted conducting the experiments. All authors read and approved the final manuscript

Ethics approval and consent to participate

Not applicable.

\section{Consent for publication}

Not applicable.

\section{Competing interests}

The authors have declared that no competing interests exist.

\section{Publisher's Note}

Springer Nature remains neutral with regard to jurisdictional claims in published maps and institutional affiliations.

Received: 13 December 2017 Accepted: 8 February 2018

Published online: 15 February 2018

\section{References}

1. Spaeth, M.L., Manes, K.R., Widmayer, C.C., Williams, W., Whitman, P.A., Henesian, M.: The National Ignition Facility Wavefront Requirements and optical architecture. Proc. SPIE. 5341, 25-42 (2004)

2. $Y u, G ., L i, H .$, Walker, D.D.: Removal of mid spatial-frequency features in mirror segments. J. Eur. Opt. Soc. 6, 11044 (2011)

3. Glatzel, H., Ashworth, D., Bremer, M., Chin, R., Cummings, K., Girard, L., Goldstein, M., Gullikson, E., Hudyma, R., Kennon, J., Kestner, B., Marchetti, L., Naulleau, P., Soufli, R., Spiller, E.: Projection optics for extreme ultraviolet lithography (EUVL) micro-field exposure tools (METs) with a numerical aperture of 0.5. Proc of. SPIE. 8679, 867917 (2013)

4. Shafrir, S.N., Lambropoulos, J.C., Jacobs, S.D.: A magnetorheological polishing-based approach for studying precision microground surfaces of tungsten carbides. Precis. Eng. 31(2), 83-93 (2007)

5. Arnold, T., Pietag, F.: lon beam figuring machine for ultra-precision silicon spheres correction. Precis. Eng. 41, 119-125 (2015)

6. Arnold, T., Boehm, G., Paetzelt, H.: New freeform manufacturing chain based on atmospheric plasma jet machining. J. Eur. Opt. Soc. 11, 16002 (2016)

7. Walker, D.D., Brooks, D., King, A., Freeman, R., Morton, R., McCavana, G., Kim, S.: The 'precessions' tooling for polishing and figuring flat, spherical and aspheric surfaces. Opt. Express. 11(8), 958-964 (2003)

8. Li, H.Y., Yu, G.Y., Walker, D.D., Evans, R.: Modelling and measurement of polishing tool influence. J. Eur. Opt. Soc. 6, 11048 (2011)

9. Walker, D.D., Yu, G., Bibby, M., Dunn, C., Li, H., Wu, H.Y., Zheng, X., Zhang, P.: Robotic automation in computer controlled polishing. J. Eur. Opt. Soc. 11, 16005 (2016)

10. Feit, M.D., Rubenchik, A.M.: Influence of subsurface cracks on laser induced surface damage. Proc. SPIE. 5273, 264-272 (2004)

11. Lawson, J.K., Wolfe, C.R., Manes, K.R., Trenholme, J.B., Aikens, D.M., English Jr., R.E.: Specification of optical components using the power spectral density function. Proc of. SPIE. 2536, 38-50 (1995)
12. Pohl, M., Börret, R.: Simulation of mid-spatials from the grinding process. J. Eur. Opt. Soc. 11, 16010 (2016)

13. Wang, C.J., Yang, W., Ye, S., Wang, Z.Z., Zhong, B., Guo, Y.B., Xu, Q.: Optimization of parameters for bonnet polishing based on the minimum residual error method. Opt. Eng. 53(7), 075108 (2014)

14. Wang, C., Yang, W., Ye, S., Wang, Z.Z., Yang, P., Peng, Y., Guo, Y., Xu, Q.: Restraint of tool path ripple based on the optimization of tool step size for sub-aperture deterministic polishing. Int. J. Adv. Manuf. Technol. 75, 1431-1438 (2014)

15. Wang, C.J., Wang, Z., Xu, Q.: Unicursal random maze tool path for computercontrolled optical surfacing. Appl. Opt. 54(34), 10128-10136 (2015)

16. Wang, T., Cheng, H.B., Yang, H., Wu, W.T., Tam, H.Y.: Controlling mid-spatial frequency errors in magnetorheological jet polishing with a simple vertical model. Appl. Opt. 54(21), 6433-6440 (2015)

17. Tam, H.Y., Cheng, H.B., Dong, Z.C.: Peano-like paths for subaperture polishing of optical aspherical surfaces. International journal of advanced manufacturing technology. Appl. Opt. 52(15), 3624-3636 (2015)

18. Tam, H.Y., Cheng, H.B.: An investigation of the effects of the tool path on the removal of material in polishing. J. Mater. Process. Technol. 210, 807-818 (2010)

19. Khakpour, H., Birglen, L., Tahan, S.: Uniform scanning path generation for abrasive waterjet polishing of free-form surfaces modeled by triangulated meshes. Int. J. Adv. Manuf. Technol. 77, 1167-1176 (2015)

20. Dunn, C.R., Walker, D.D.: Pseudo-random tool paths for CNC sub-aperture polishing and other applications. Opt. Express. 16(23), 18942-18949 (2008)

21. Hu, H., Dai, Y.F., Peng, X.Q.: Restraint of tool path ripple based on surface error distribution and process parameters in deterministic finishing. Opt. Express. 18(22), 22973-22981 (2010)

22. Dai, Y.F., Shi, F., Peng, X.Q., Li, S.Y.: Restraint of mid-spatial frequency error in magneto-rheological finishing (MRF) process by maximum entropy method. Sci. China Ser. E: Technol. Sci. 52(10), 3092-3097 (2009)

\section{Submit your manuscript to a SpringerOpen ${ }^{\circ}$ journal and benefit from:}

- Convenient online submission

- Rigorous peer review

- Open access: articles freely available online

- High visibility within the field

- Retaining the copyright to your article

Submit your next manuscript at $>$ springeropen.com 